\title{
Hematoma de tabique: informe de dos casos pediátricos
}

\author{
Septal hematoma: Report of two pediatric cases
}

\author{
Dra. Malena Vega ${ }^{a}$, Dra. Florencia Bianchi ${ }^{a}$ Dr. Daniel Cruz y Dra. Roxana Spini ${ }^{b}$
}

\begin{abstract}
RESUMEN
Los hematomas y abscesos septales constituyen una urgencia en rinología y requieren tratamiento quirúrgico temprano debido al riesgo de complicaciones infecciosas, funcionales y estéticas. Suelen aparecer como consecuencia deun traumatismo nasal, aunque también se han descrito en relación con otros desencadenantes. La acumulación de sangre o pus entre el mucopericondrio y el cartílago septal causa lesión por necrosis avascular en el cartílago y la destrucción de este.

Desde el punto de vista clínico, se presenta como insuficiencia ventilatoria nasal y dolor facial. Con menor frecuencia, el motivo de consulta es deformidad del dorso nasal, epistaxis, rinorrea purulenta y fiebre.

Se presentan en este trabajo dos casos clínicos con diagnóstico de hematoma septal que requirieron cirugía.

Palabras clave: tabique nasal, hematoma, absceso, heridas y traumatismos, niño.
\end{abstract}

\section{ABSTRACT}

Hematomas and septal abscesses are an emergency in rhinology and require early surgical treatment due to the risk of infectious, functional, and aesthetic complications. They generally occur as a consequence of nasal trauma, although they have also been described in relation to other triggers. Accumulation of blood or pus between the mucoperichondrium and septal cartilage, causes avascular necrosis injury to the cartilage with cartilage destruction.

Clinically it presents as nasal ventilatory insufficiency and facial pain. In a lower percentage, the reason for consultation was nasal dorsal deformity, epistaxis, purulent rhinorrhea and fever. We present two clinical cases with septal hematoma. Both required surgery.

Key words: nasal septum, hematoma, abscess, wounds and injuries, child.

http: / / dx.doi.org/10.5546/ aap.2021.e261

Cómo citar: Vega M, Bianchi F. Cruz D, Spini R. Hematoma de tabique: informe de dos casos pediátricos. Arch Argent Pediatr 2021; 119(3):e261-e263.

a. Residencia de Otorrinolaringología Infantil.

b. División de Otorrinolaringología.

Hospital General de Niños "Pedro de Elizalde", Ciudad

Autónoma de Buenos Aires, Argentina.

Correspondencia:

Dra. Malena Vega: malena.v.martari@gmail.com

Financiamiento: Ninguno.

Conflicto de intereses: Ninguno que declarar.

Recibido: 6-8-2020

Aceptado: 15-1-2021

\section{INTRODUCCIÓN}

Los hematomas (HS) y los abscesos septales (AS) son complicaciones frecuentes de los traumatismos nasales, y también pueden aparecer en el período posquirúrgico de cirugías nasales, en forunculosis nasal y en procesos infecciosos dentarios. Son poco comunes en la población pediátrica. ${ }^{1}$ Según la literatura, aproximadamente el $0,8 \%$ de los casos de traumatismos se complica con un AS y un $1,6 \%$ con un HS. ${ }^{2}$ En muchos casos, no se encuentra antecedente de traumatismo. ${ }^{1}$

Tanto los HS como los AS se caracterizan por la presencia de sangre o pus en el espacio subpericóndrico, que compromete la irrigación del cartílago septal. Es necesario realizar un diagnóstico temprano con el fin de evitar complicaciones en el corto y el mediano plazo.

El objetivo es minimizar las comorbilidades. Por lo tanto, es menester concientizar a los especialistas en pediatría o en el área de emergencias, con el fin de aumentar el índice de sospecha y la derivación oportuna a un otorrinolaringólogo. En este trabajo se presentan dos casos clínicos de HS que requirieron resolución quirúrgica.

\section{CASO CLÍNICO Paciente 1}

Niña de 5 años, previamente sana, concurre al servicio de otorrinolaringología por insuficiencia ventilatoria nasal (IVN) bilateral de un mes de evolución. Había consultado antes en otros centros por fiebre y rinorrea, donde se le habían indicado lavajes nasales hidrosalinos y amoxicilina. Ante la persistencia de fiebre y la aparición de IVN, se rota el antibiótico a amoxicilina-ácido clavúlanico en dosis de $50 \mathrm{mg} / \mathrm{kg} /$ día y se solicita interconsulta con otorrinolaringología, a la cual concurre 15 días después.

Al examen físico se constatan fauces y otoscopia normal. En la rinoscopia con óptica de $30^{\circ}$, se observa abombamiento bilateral del tabique nasal (Figura $1 A$ y Video). En la inspección del perfil se observa la nariz con deformidad en silla de montar por derrumbamiento de la 
pirámide cartilaginosa (Figura 1B). Los padres no refieren antecedentes de traumatismo nasal. Con diagnóstico de AS/HS, se indica drenaje quirúrgico urgente.

Se realiza punción y aspiración de abundante material hematopurulento que se envía a cultivo y una incisión del tabique membranoso en forma bilateral, con desbridamiento y lavaje de la cavidad. Se coloca drenaje de goma y taponaje. La niña permanece internada con ceftriaxona (50 mg/ kg/día) + (clindamicina $30 \mathrm{mg} / \mathrm{kg} /$ día) por vía intravenosa (IV). El cultivo es positivo para Staphylococcus aureus resistente a la meticilina. A las 72 horas se retira el taponaje, la niña tiene buena evolución clínica y se externa con indicación de trimetroprima-sulfametozaxol (10 mg/kg/día); cumple tratamiento antibiótico de 14 días en total, con resolución completa del hematoma (Figura 1C y Vídeo).

\section{Paciente 2}

Niña de 7 años, previamente sana, consulta por IVN bilateral de cuatro días de evolución. Se encuentra afebril y refiere antecedente de traumatismo hace 13 días.

Al examen físico, se constatan fauces y oídos normales. En la rinoscopia con óptica de $30^{\circ}$, se observa abombamiento bilateral del tabique nasal sin secreción en las fosas nasales (Figura 1D y Vídeo). Con diagnóstico de AS/HS, se indica drenaje quirúrgico urgente.

Se realiza punción y aspiración de escaso material hematopurulento, que se envía a cultivo. Luego, se realiza una incisión del tabique cartilaginoso en la fosa nasal derecha. Se desbrida y se colocan un drenaje de goma y taponaje bilateral. Se indica amoxicilina-ácido clavulánico en dosis de $50 \mathrm{mg} / \mathrm{kg} /$ día en forma ambulatoria. A las 72 horas, se retira el taponaje, con buena

FIGURA 1. A: hematoma septal bilateral visto con rinoscopia anterior, B: deformidad en silla de montar en la inspección de perfil; C: resolución del hematoma vista con rinoscopia anterior 72 horas después de la cirugía, D: hematoma septal bilateral visto con rinoscopia anterior. (A-C: caso clínico 1; D: caso clínico 2).
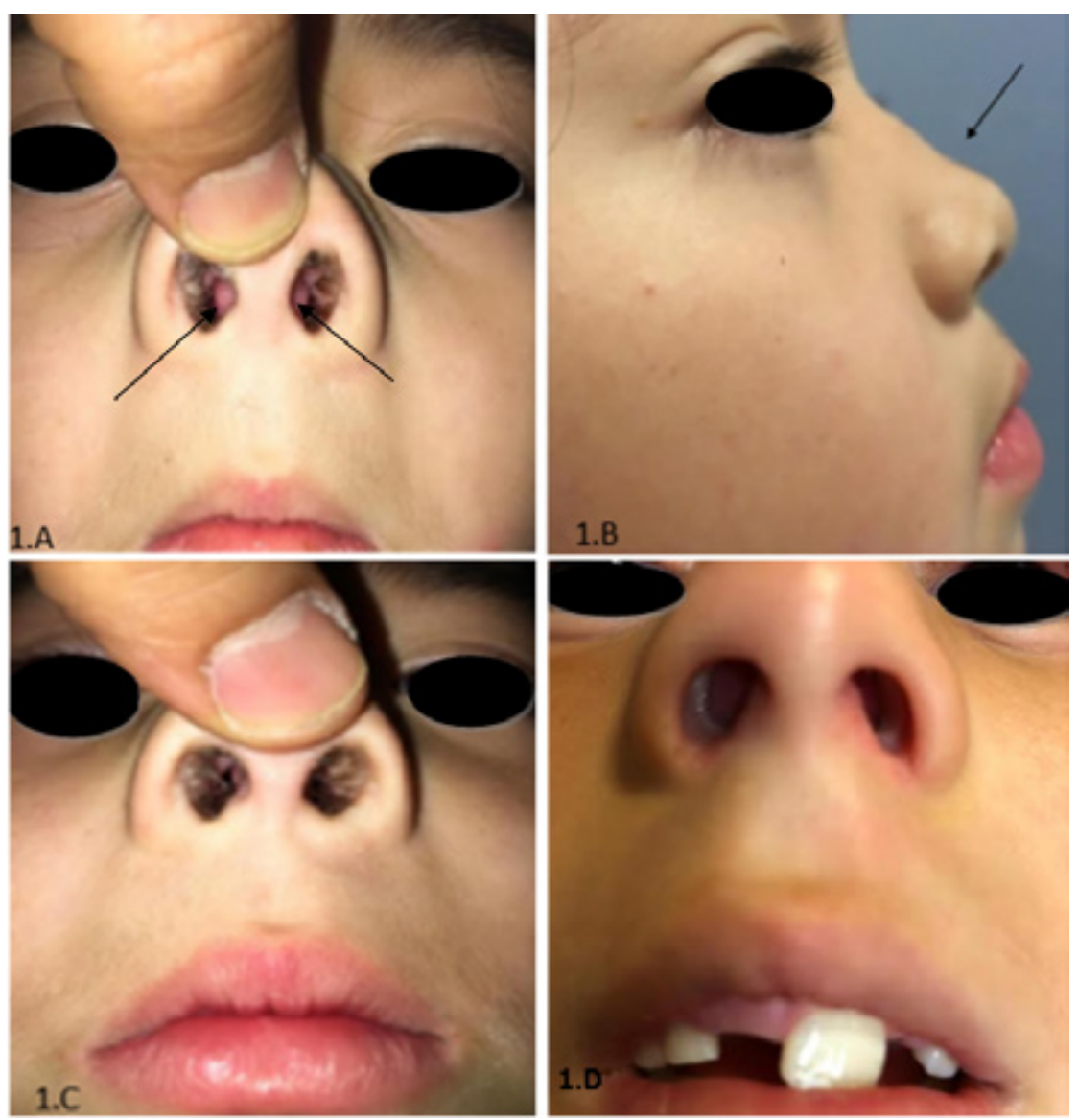
evolución clínica. En el cultivo se aísla Haemofilus influenzae betalactamasa negativo. La paciente cumplió tratamiento antibiótico durante 10 días, con resolución total del hematoma.

\section{DISCUSIÓN}

El cartílago septal se nutre por un mecanismo de imbibición a partir de los vasos sanguíneos del mucopericondrio. Los traumatismos nasales desencadenan la rotura de los vasos sanguíneos con acumulación de sangre entre el mucopericondrio y el cartílago, lo que da como resultado necrosis avascular y licuefacción de este último. ${ }^{3} \mathrm{El}$ daño comienza a las 24 horas y la necrosis se consolida pasadas las 72 horas del traumatismo. ${ }^{4}$ Por este motivo, se debe citar a control al paciente pasado este período, para excluir la formación tardía de hematomas.

La IVN es el principal motivo de consulta en pacientes con HS. ${ }^{5}$ El $50 \%$ de los pacientes presentan dolor facial y, en menor porcentaje, deformidad del dorso nasal, epistaxis, rinorrea purulenta y fiebre. ${ }^{6}$

El diagnóstico es clínico, pero requiere de un examen físico minucioso y sistemático. ${ }^{7}$ Puede realizarse con otoscopia, con un espéculo de nariz más luz frontal y/o con un endoscopio rígido o flexible. La maniobra de levantamiento de la punta nasal suele causar dolor. Se identifica al HS como un abultamiento unilateral o bilateral ${ }^{1}$ (sobre todo con fractura concomitante), que no desaparece frente a la topicación local con vasoconstrictores y que fluctúa a la palpación con un elemento romo como un portaalgodón. ${ }^{5}$

El tratamiento es quirúrgico. Se comienza con una punción-aspiración con aguja y la muestra se remite para cultivo bacteriológico. ${ }^{8}$ Luego se realiza una incisión de la mucosa en L o transversal de Killian ${ }^{I}$, lavaje con solución fisiológica y desbridamiento. ${ }^{4} \mathrm{Si}$ bien algunos trabajos lo cuestionan por el riesgo de sobreinfección, varios autores recomiendan colocar un drenaje de tipo Penrose. ${ }^{3,5}$ Luego del drenaje, y con el fin de aproximar la mucosa al cartílago septal, la realización de puntos transfixiantes con sutura reabsorbible

VíDEO. Rinoscopía con óptica $30^{\circ}$ evitan el espacio muerto y, de esta manera, la reacumulación del hematoma. ${ }^{4}$ Se describe la realización del taponaje nasal con gasas embebidas en vaselina líquida o con tapones nasales. Se prefieren estos últimos por ser más confortables para el paciente y más fáciles de colocar y retirar. ${ }^{1}$ Se indica tratamiento antibiótico empírico con ampicilina-sulbactam, ${ }^{6}$ para evitar sobreinfecciones por $S$. aureus, $H$. influenzae $y$ Streptococco pneumoniae. El taponaje se retira a las 48-72 horas. $^{8}$

Las complicaciones posquirúrgicas tempranas son la recidiva, el seroma o la infección. ${ }^{9}$ En el largo plazo, las complicaciones son principalmente estéticas y funcionales, como deformidad del dorso nasal en silla de montar, rotación hacia arriba de la punta nasal e hipoplasia o retroposición del tercio medio de la cara. ${ }^{4}$ Estas últimas pueden aparecer hasta por lo menos 12 meses después. ${ }^{10}$ En el caso de la primera paciente, presentaba nariz en silla de montar al momento de la consulta por HS de larga data, sin tener la certeza de traumatismo nasal como antecedente.

Los HS y AS son complicaciones que requieren tratamiento quirúrgico urgente. El reconocimiento temprano y el tratamiento oportuno son clave para prevenir complicaciones infecciosas, trastornos funcionales permanentes y deformidades estéticas.

\section{REFERENCIAS}

1. SavageR, Valvich C. Hematoma of the nasal septum. Pediatr Rev. 2006; 27(12):478-9.

2. Sayin I, Yazici ZM, Boozkurt E, Kayhan FT. Nasal Septal Hematoma and Abscess in Children. JCraniofac Surg. 2011; 22(6):e17-9.

3. Alvarez H, Osorio J, De Diego JI, Prim MP, et al. Sequelae after nasal septum injuries in children. Auris Nasus Larynx. 2000; 27(4):339-42.

4. Jan Menger D, Tabink I, Nolst Trenité G. Treatment of Septal Hematomas and Abscesses in Children. Facial Plast Surg. 2007; 23(4):239-42.

5. Puricelli M, Zitsch RP 3rd. Septal hematoma following nasal trauma. J Emerg Med. 2016; 50(1):121-2.

6. Henry M, Hern HG. Traumatic Injuries of the Ear, Nose and Throat. Emerg Med Clin North Am. 2019; 37(1):131-6.

7. Landis BN, Borner U. Septal Hematoma: Always Think About. J Pediatr. 2013; 163(4):1223.

8. Canty PA, Berkowitz RG. Hematoma and abscess of the nasal septum in children. Arch Otolaryngol Head Neck Surg. 1996; 122(12):1373-6.

9. Toback S. Nasal septal hematoma in an 11-month-old infant: a case report and review of the literature. Pediatr Emerg Care. 2013; 19(4):265-7.

10. Kass JI, Ferguson BJ. Treatment of hematoma of the nasal septum. N Engl J Med. 2015; 372:e28. 